\title{
Serum CA242: a biomarker for diagnosis, progression, and metastasis in multiple tumors
}

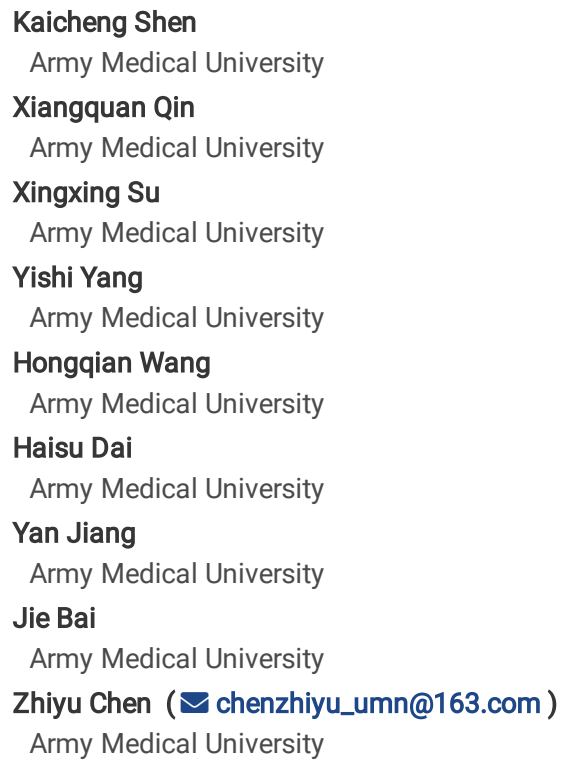




\section{Abstract \\ Background}

CA242 is a classic biomarker used for diagnosing digestive tract tumors, especially pancreatic cancer. However, CA242 serum levels in some tumor patients and what might influence these levels remain unknown or uncertain. This study aimed to reveal the pancancer landscape of serum CA242 levels and identify some influencing factors.

\section{Methods}

In the current study, serum CA242 levels and clinical information, including clinical stage and metastatic status, were collected from 37,493 patients with 35 different types of neoplastic disease, and CA242 values were also obtained for 880 healthy controls.

\section{Results}

Serum CA242 levels in patients with one of 21 different cancers were significantly higher than those in healthy controls and had diagnostic value in 9 tumors $(A U C>0.7, P<0.05)$; serum CA242 levels were increased across all malignant digestive tract tumors. Compared to patients without metastasis, cancer patients with one of 15 kinds of cancer with lymph node metastasis and distant metastasis showed elevated CA242 levels. Moreover, CA242 was helpful in distinguishing the clinical stage and metastatic status in 6 cancers. In addition, the presence of multiple metastases and liver metastasis might result in increased CA242 levels.

\section{Conclusions}

Overall, CA242 can not only serve as a diagnostic biomarker for malignant tumors in the digestive system but also predict the progression, stage, and metastasis of many other tumors that have not received clinical attention.

\section{Background}

Currently, cancer is one of the most critical public health problems worldwide[1].To improve therapeutic outcomes, identifying cancer patients in an early stage or precisely diagnosing the clinical stage and metastatic status is essential. Therefore, finding useful biomarkers is crucial.

CA242 is a sialylated carbohydrate antigen that was isolated in 1985[2]; it attaches to core proteins/lipids detected on the cell surface or in serum[3]. Subsequently, the serum level of CA242 was reported to be increased in tumor patients with pancreatic cancer[4-8]. CA242 was found to be overexpressed more strongly in pancreatic carcinoma cells than in adjacent tissues[4]. Moreover, its expression correlates with the degree of differentiation and clinical stage[4, 9]; however, CA242 tumor expression does not have any relationship with serum levels[4]. Interestingly, the serum CA242 level is scarcely affected by cholestasis and benign obstructive jaundice, while its sensitivity and specificity are similar to those of CA19-9 and CA50[7]. Hence, it is believed that serum CA242 is a product of cancer cells[10,11].

Currently, although CA242 has been used as a first-line clinical marker of pancreatic cancer for more than a decade[11], research on it has continued. CA242 is not merely a diagnostic predictor[12,13] but also participates in forecasting the progression and prognosis of many tumors[14-16]. In pancreatic cancer, colorectal cancer[17-19], gastric cancer[20, 21] and other tumors and when used alone or in combination with CA19-9, CA50, CEA and other biomarkers[4, 7, $14,22-24]$, CA242 is gradually becoming better understood and applied more broadly. Therefore, CA242 is routinely included in screening and monitoring many different cancers.

However, there are few comprehensive in-depth studies on the pancancer CA242 atlas across a variety of tumors. Except for in pancreatic cancer, colorectal cancer and some other tumors, we have no understanding of the potential of the serum CA242 level in patients with tumors, and no systematic comparison has been performed until now. Therefore, we conducted a retrospective study of serum CA242 levels in 37,493 patients with 35 different defined neoplastic diseases and 880 healthy controls who visited the clinical laboratory of our hospital in the past five years.

\section{Methods}

Patients

All data for the detection of CA242 in the past five years were collected from the Medical Big Data and Artificial Intelligence Center of SouthWest Hospital, the First Affiliated Hospital of the Army Medical University. Serum CA242 values for 37,493 patients suffering from 35 benign or malignant tumors and 880 healthy controls were measured and analyzed. All patients were definitively diagnosed, and in some patients, the extent of the tumor, including the lymph node metastasis (LNM) status, distant metastasis (DM) status, and TNM staging according to the American Joint Commission on Cancer (AJCC), was recorded. Rapid ethical approval was acquired, and all methods followed the relevant guidelines and regulations.

Assays 
The detection of serum CA242 in all patients was performed by the Laboratory Department of the First Affiliated Hospital of the Army Medical University. Serum CA242 levels were quantified by the TESMI ${ }^{\text {m }}$ Tumor Marker Panel (7 markers) (flow cytometry fluorescence method) (Shanghai Tellgen Cooperation, Shanghai, China) according to the manufacturer's instructions. The technical platform and instrument were the Tellgen Super Multiplex Immunoassay (TESMI) F3999 system. The cutoff value for serum tumor markers recommended by the kit was $20 \mathrm{U} / \mathrm{mL}$ for CA242.

\section{Statistical analysis}

All data were processed with Microsoft Excel (Microsoft, Inc., USA). All the original data were imported, cleaned, and collated by MySQL version 8.0.15 (Oracle Corporation), Navicat version 12.0.29, and Python version 3.7.2 (Python Software Foundation). Statistical analysis was performed by using SPSS version 23. Due to the data having a nonnormal distribution, the Kruskal-Wallis test was used for statistical analysis, and Dunn's multiple-comparisons test was used for performing multigroup comparisons. Enumerative data were compared with the chi-square test. The prediction value was calculated by receiver operating characteristic (ROC) curve analysis. Then, the area under the curve (AUC) was calculated, and the largest value of the Youden index was chosen as the cut off. All tests were two-tailed, and $\mathrm{P}<0.05$ was considered statistically significant.

\section{Results}

\section{Patient characteristics}

The serum CA242 levels of 37,493 patients with 35 types of tumors and 880 healthy controls were statistically compared and analyzed, and the results are shown in Table 1. To visualize the results, we constructed Fig. 1 and ranked all the diseases from highest to lowest according to the median CA242 level. 
Table 1

Characteristics and serum CA242 levels of patients and healthy $\mathrm{C}$

\begin{tabular}{|c|c|c|c|c|c|c|c|c|c|c|c|}
\hline & & Count & & Age & & & & & & & RC \\
\hline & Case & Female & Male & Female & Male & $p$ value & Mean(SD) & Medin(25\%,75\%) & $\begin{array}{l}P \\
\text { value }\end{array}$ & Fold change & $\mathrm{Al}$ \\
\hline Total & 38373 & 18618 & 19755 & 49.76 & 44.11 & 0.35 & 21.143(37.53) & $8.06(4.05,33.95)$ & - & - & - \\
\hline Healthy control & 880 & 397 & 483 & 57.86 & 55.98 & 0.08533 & $5.58(4.56)$ & $4.32(2.46,7.26)$ & - & - & - \\
\hline Pancreatic cancer & 650 & 260 & 390 & 60.06 & 60.65 & 0.52500 & $94.28(120.54)$ & $35.08(8.51,142.23)$ & $<0.0001$ & 8.12037037 & $\begin{array}{l}0 .\} \\
0 .\}\end{array}$ \\
\hline $\begin{array}{l}\text { Gallbladder } \\
\text { carcinoma }\end{array}$ & 193 & 125 & 68 & 60.18 & 60.90 & 0.67000 & 78.64(113.50) & $24.94(6.94,98.67)$ & $<0.0001$ & 5.773148148 & $\begin{array}{l}0 .\} \\
0 .\}\end{array}$ \\
\hline Cholangiocarcinoma & 856 & 355 & 501 & 62.46 & 62.70 & 0.77600 & 77.22(117.85) & $20.68(7.18,85.93)$ & $\begin{array}{l}< \\
0.0001\end{array}$ & 4.787037037 & $\begin{array}{l}0 . \xi \\
0 .\}\end{array}$ \\
\hline Colorectal cancer & 4486 & 1677 & 2809 & 59.84 & 61.18 & 0.00100 & $26.50(59.58)$ & $8.39(4.78,497.4)$ & $<0.0001$ & 1.940972222 & $\begin{array}{l}0 . \\
0 .\end{array}$ \\
\hline Mediastinal Cancer & 8 & 0 & 8 & 0.00 & 55.80 & - & 41.97(88.92) & $12(8.95,15.34$ & 0.0777 & 2.777777778 & $\begin{array}{l}0 .\} \\
0\}\end{array}$ \\
\hline $\begin{array}{l}\text { Periampullary } \\
\text { carcinoma }\end{array}$ & 83 & 31 & 52 & 57.45 & 57.88 & 0.87500 & 47.87(93.24) & $11.66(4.21,36.35)$ & $<0.0001$ & 2.699074074 & $\begin{array}{l}0 .{ }^{-} \\
0 . \Omega^{\prime}\end{array}$ \\
\hline Testicular cancer & 16 & 0 & 16 & 0.00 & 52.60 & - & $36.24(93.67)$ & $9.01(4.69,21.81)$ & 0.0772 & 2.085648148 & $\begin{array}{l}0 . \\
0.8\end{array}$ \\
\hline Ovarian cancer & 1211 & 1211 & 0 & 51.17 & 0.00 & - & $19.52(42.27)$ & $8.12(4.4,16.13)$ & $<0.0001$ & 1.87962963 & $\begin{array}{l}0 .: \\
0 .\end{array}$ \\
\hline Vaginal cancer & 24 & 24 & 0 & 52.67 & 0.00 & - & $21.19(44.67)$ & $8.11(5.38,14.67)$ & 0.0012 & 1.876157407 & $\begin{array}{l}0 .{ }^{-} \\
0 . \varepsilon^{\prime}\end{array}$ \\
\hline Ureter cancer & 12 & 12 & 0 & 61.80 & 0.00 & - & $28.59(42.75)$ & $8.05(4.74,26.99)$ & 0.1396 & 1.862268519 & $\begin{array}{l}0 . \\
0\}\end{array}$ \\
\hline $\begin{array}{l}\text { Neuroendocrine } \\
\text { carcinoma }\end{array}$ & 25 & 14 & 11 & 49.36 & 58.45 & 0.06000 & $12.16(22.90)$ & 7.33(3.87,10.02) & 0.4501 & 1.696759259 & $\begin{array}{l}0 . t_{1} \\
0 .\end{array}$ \\
\hline Gastric cancer & 2015 & 635 & 1380 & 57.28 & 61.39 & $<.0001$ & $28.03(66.02)$ & $7.26(4.07,15.54)$ & $<0.0001$ & 1.680555556 & $\begin{array}{l}0 . \ell \\
0 . l_{1}\end{array}$ \\
\hline $\begin{array}{l}\text { Nasonasopharyngeal } \\
\text { carcinoma }\end{array}$ & 811 & 219 & 592 & 49.30 & 51.46 & 0.00900 & $11.05(16.97)$ & $7.05(4.02,12)$ & $<0.0001$ & 1.631944444 & $\begin{array}{l}0.6 \\
0.6\end{array}$ \\
\hline $\begin{array}{l}\text { Gastric cardia } \\
\text { adenocarcinoma }\end{array}$ & 24 & 5 & 19 & 64.40 & 65.89 & 0.80400 & $36.25(60.54)$ & $7.045(4.23,23.92)$ & 0.0012 & 1.876157407 & $\begin{array}{l}0 .{ }^{-} \\
0 . \xi\end{array}$ \\
\hline $\begin{array}{l}\text { Craniofacial } \\
\text { malignant tumour }\end{array}$ & 395 & 112 & 283 & 57.83 & 59.21 & 0.32500 & $10.10(19.92)$ & $6.68(3.77,10.11)$ & $\begin{array}{l}< \\
0.0001\end{array}$ & 1.546296296 & $\begin{array}{l}0.6 \\
0 . l_{1}\end{array}$ \\
\hline Cervical cancer & 2169 & 2169 & 0 & 51.29 & 0.00 & - & $11.54(23.97)$ & $6.62(4.02,11.58)$ & $<.0001$ & 1.532407407 & $\begin{array}{l}0.6 \\
0.1\end{array}$ \\
\hline Endometrial cancer & 438 & 438 & 0 & 55.63 & 0.00 & - & $11.70(17.66)$ & $6.59(3.95,11.47)$ & $\begin{array}{l}< \\
0.0001\end{array}$ & 1.525462963 & $\begin{array}{l}0.6 \\
0.6\end{array}$ \\
\hline Lung cancer & 7536 & 2538 & 4998 & 59.78 & 62.36 & $<0.0001$ & $18.49(45.97)$ & $6.48(3.67,12.9)$ & $<0.0001$ & 1.5 & $\begin{array}{l}0.6 \\
0.6\end{array}$ \\
\hline Breast cancer & 3824 & 3824 & 0 & 50.94 & 0.00 & - & 9.57(16.15) & $6.24(3.72,10.51)$ & $<0.0001$ & 1.443287037 & $\begin{array}{l}0.6 \\
0.6\end{array}$ \\
\hline Vulvar cancer & 52 & 52 & 0 & 53.05 & 0.00 & - & $7.82(6.38)$ & $6.17(3.69,11.19)$ & 0.2174 & 1.428240741 & 0.6 \\
\hline $\begin{array}{l}\text { Fallopian Tube } \\
\text { Cancer }\end{array}$ & 10 & 10 & 0 & 61.80 & 0.00 & - & $7.01(4.62)$ & $6.01(3.65,10.15)$ & > 0.9999 & 1.391203704 & $\begin{array}{l}0.6 \\
0 .\end{array}$ \\
\hline Renal Cell Cancers & 902 & 333 & 569 & 57.80 & 58.40 & 0.51800 & $12.93(38.40)$ & $5.39(3.13,9.98)$ & $\begin{array}{l}< \\
0.0001\end{array}$ & 1.246527778 & $\begin{array}{l}0 . ! \\
0.1\end{array}$ \\
\hline Laryngocarcinoma & 333 & 24 & 309 & 58.75 & 62.48 & 0.08300 & $6.79(6.35)$ & $5.29(2.91,8.85)$ & 0.1694 & 1.224537037 & 0.1 \\
\hline Lymphoma & 33 & 16 & 17 & 50.69 & 55.76 & 0.37300 & $8.91(12.53)$ & $5.25(3.12,10.09)$ & > 0.9999 & 1.215277778 & $\begin{array}{l}0 . ! \\
0.6\end{array}$ \\
\hline
\end{tabular}




\begin{tabular}{|c|c|c|c|c|c|c|c|c|c|c|c|}
\hline & & Count & & Age & & & & & & & $\mathrm{RC}$ \\
\hline Liver cancer & 4347 & 711 & 3636 & 56.76 & 55.31 & 0.00400 & $17.09(47.40)$ & $5.22(3.15,9.785)$ & $<.0001$ & 1.208333333 & $\begin{array}{l}0.1 \\
0.1\end{array}$ \\
\hline Esophagus cancer & 1328 & 272 & 1056 & 64.05 & 63.88 & 0.76600 & 10.95(35.79) & $5.14(3.15,8.66)$ & $<0.0001$ & 1.188657407 & $\begin{array}{l}0 . ! \\
0 . !\end{array}$ \\
\hline Prostatic cancer & 727 & 0 & 727 & 0.00 & 73.06 & - & $9.27(20.47)$ & $5.12(3.16,8.94)$ & $<0.0001$ & 1.185185185 & $\begin{array}{l}0 . ! \\
0.1\end{array}$ \\
\hline Skin cancer & 194 & 91 & 103 & 65.09 & 62.47 & 0.16800 & $6.18(4.65)$ & $5.02(3.20,7.85)$ & > 0.9999 & 1.162037037 & $\begin{array}{l}0 . ! \\
0.1\end{array}$ \\
\hline Bladder cancer & 602 & 114 & 488 & 62.75 & 66.49 & 0.00300 & $9.26(21.00)$ & $4.83(3.07,8.28)$ & 0.0225 & 1.118055556 & 0.1 \\
\hline Thyroid cancer & 3477 & 2625 & 852 & 45.72 & 45.28 & 0.38700 & $6.83(10.67)$ & $4.79(2.93,8.07)$ & 0.0143 & 1.108796296 & $\begin{array}{l}0 . ! \\
0 . !\end{array}$ \\
\hline $\begin{array}{l}\text { Cervical } \\
\text { intraepithelial } \\
\text { neoplasias }\end{array}$ & 107 & 107 & 0 & 45.19 & 0.00 & - & $6.19(4.81)$ & $4.74(2.94,8.15)$ & > 0.9999 & 1.097222222 & $\begin{array}{l}0 . ! \\
0 . !\end{array}$ \\
\hline Penile cancer & 65 & 0 & 65 & 0.00 & 56.40 & - & $5.3(4.41)$ & $4.01(2.51,7.1)$ & > 0.9999 & 0.928240741 & $\begin{array}{l}0 . ! \\
0 . !\end{array}$ \\
\hline Leukemia & 504 & 206 & 298 & 41.97 & 43.59 & 0.33200 & $6.32(7.33)$ & $4(2.37,7.68)$ & $\begin{array}{l}> \\
0.9999\end{array}$ & 0.925925926 & $\begin{array}{l}0 . ! \\
0 . !\end{array}$ \\
\hline $\begin{array}{l}\text { Benign colorectal } \\
\text { neoplasms }\end{array}$ & 21 & 7 & 14 & 62.86 & 58.29 & 0.44800 & 7.10(8.33) & $3.85(2.70,8.22)$ & > 0.9999 & 0.891203704 & $\begin{array}{l}0.1 \\
0.1\end{array}$ \\
\hline Thymic cancer & 15 & 4 & 11 & 45.75 & 60.09 & 0.08600 & $6.54(6.31)$ & $3.58(2.70,8.46)$ & $\begin{array}{l}> \\
0.9999\end{array}$ & 0.828703704 & $\begin{array}{l}0 . ! \\
0.1\end{array}$ \\
\hline
\end{tabular}

SD standard deviation

The mean and median serum CA242 levels of most patients with malignant disease were significantly higher than those of the healthy controls, and the serum CA242 levels of all the patients with malignant digestive tract malignant tumors were increased. Compared to the healthy controls, the tumor patients with the most significantly increased median levels were those with pancreatic cancer (8.12-fold), gallbladder cancer (5.77-fold), or cholangiocarcinoma (4.78fold). However, the patients with fallopian tube cancer, lymphoma, penile cancer, leukemia, benign colorectal neoplasms, or thymic cancer were not significantly different from the healthy controls (Fig. 1 and Fig. 2).

To test the capacity of CA242 to diagnose tumors, we made ROC curves for all the diseases. The cancers with a value greater than 0.8 were pancreatic cancer (0.8616), cholangiocarcinoma (0.8349), and gallbladder carcinoma (0.8145). In addition, the AUCs of colorectal cancer (0.7268), testicular cancer (0.7165), ovarian cancer (0.7053), vaginal cancer (0.7604), ureteral cancer (0.7323) and cardiac cancer (0.7604) were all greater than 0.7(Table 1).

\section{CA242 in different metastatic situations}

Serum CA242 levels varied widely in most diseases; for example, the median value for colorectal cancer was 8.39 , but the quartile range was from 4.78 to 497.4 (Table 1 and Fig. 1). We speculated that a factor such as the progression of the disease might affect the levels, so we divided the tumor patients into three groups according to their metastatic situation: no metastasis (NM), LNM, and DM.

We found that the mean and median serum CA242 levels were increased in most tumor patients who exhibited LNM or DM compared to those who exhibited NM. The diseases that showed the most significant increases in the serum CA242 level were cholangiocarcinoma [median (quartile) for NM: 17.79 (6.77, 65.95), LNM: 76.03 (16.39, 204.02), and DM: 34.01 (12.24, 120.94)]; gallbladder carcinoma [NM: 8.125 (3.66, 37.28) and DM: 36.85 (11.84, 159.89)]; periampullary carcinoma [NM: $6.31(3.76,27.07)$ and DM: $19.56(11.37,110.625)]$; and pancreatic cancer [NM: 22.71 (6.97, 95.74$)$ and DM: 57.66 (17.42, 189.06)]. Additionally, endometrial cancer and colorectal cancer showed levels that increased approximately two fold, and CA242 levels increased significantly in patients with metastasized cancer, including those with lung cancer, cervical cancer, liver cancer, bladder cancer, breast cancer, esophageal cancer, or craniofacial malignant tumor. In contrast, the median in vulvar cancer patients with LNM was $3.49(3.1,4.47)$, which was significantly lower than that in patients with NM $[7.99(4.45,12.25)]$ (Table 2). 
Table 2

Serum CA242 in different metastatic situations

\begin{tabular}{|c|c|c|c|c|c|c|c|c|c|c|}
\hline & & Count & & Age & & & & p value & & ROC \\
\hline & Case & Female & Male & Female & Male & median(SD) & Median(25\%,75\%) & $\begin{array}{l}\text { Control } \\
\text { vs }\end{array}$ & NM vs. & $\begin{array}{l}\text { AUC( } 95 \% \text { confiden } \\
\text { interval) }\end{array}$ \\
\hline \multicolumn{11}{|c|}{$\begin{array}{l}\text { Nasopharyngeal } \\
\text { carcinoma }\end{array}$} \\
\hline NM & 323 & 95 & 228 & 49.98 & 52.25 & 10.94(19.18) & $6.81(3.76,11.66)$ & <.0001 & & $\begin{array}{l}0.6509(0.6154, \\
0.6865)\end{array}$ \\
\hline LNM & 323 & 92 & 231 & 48.86 & 50.30 & $11.12(12.67)$ & 7.12(4.10,13.85) & $\dot{0} 0001$ & 0.556 & $\begin{array}{l}0.6824(0.6387, \\
0.7261)\end{array}$ \\
\hline DM & 165 & 32 & 133 & 48.59 & 52.09 & $11.12(19.60)$ & 7.23(4.37,11.15) & $\begin{array}{l}<.0001 \\
0.000\end{array}$ & $\overrightarrow{0.9999}$ & $\begin{array}{l}0.6809(0.6466 \\
0.7152)\end{array}$ \\
\hline \multicolumn{11}{|c|}{ Cholangiocarcinoma } \\
\hline NM & 693 & 286 & 407 & 62.95 & 63.57 & 67.89(109.21) & $17.79(6.77,65.95)$ & $\begin{array}{l}<.0001 \\
0.000\end{array}$ & - & $\begin{array}{l}0.8198(0.7979 \\
0.8416)\end{array}$ \\
\hline LNM & 95 & 39 & 56 & 60.54 & 59.00 & $129.45(145.77)$ & $76.03(16.39,204.02)$ & $\begin{array}{l}< \\
0.0001\end{array}$ & $\begin{array}{l}<.0001 \\
0.000\end{array}$ & $0.9076(0.867,0.94$ \\
\hline DM & 68 & 30 & 38 & 60.23 & 58.82 & $99.34(137.81)$ & $34.01(12.24,120.94)$ & $\dot{0.0001}$ & 0.0409 & $\begin{array}{l}0.8882(0.8372, \\
0.9391)\end{array}$ \\
\hline \multicolumn{11}{|c|}{$\begin{array}{l}\text { Gallbladder } \\
\text { carcinoma }\end{array}$} \\
\hline NM & 88 & 55 & 33 & 60.56 & 63.45 & $44.97(67.03)$ & $14.61(5.03,58.45)$ & $\begin{array}{l}<.0001 \\
0.000\end{array}$ & - & $0.7768(0.7161,0.8$ \\
\hline LNM & 87 & 57 & 30 & 60.05 & 62.77 & 92.96(119.29) & $34.61(10.28,152.48)$ & 0.0603 & 0.6133 & $0.8584(0.802,0.91<$ \\
\hline DM & 42 & 29 & 13 & 59.13 & 59.23 & $122.87(135.63)$ & $69.285(17.85,224.68)$ & $\begin{array}{l}<.0001 \\
0.000\end{array}$ & $\begin{array}{l}<.0001 \\
0.000\end{array}$ & $0.9262(0.873,0.97$ ؟ \\
\hline \multicolumn{11}{|c|}{ Lung cancer } \\
\hline NM & 3098 & 1088 & 2010 & 60.13 & 62.43 & $11.49(26.85)$ & $5.54(3.24,9.99)$ & <. 0001 & - & $\begin{array}{l}0.5979(0.5774 \\
0.6184)\end{array}$ \\
\hline LNM & 2059 & 647 & 1412 & 60.3 & 62.48 & 15.11(36.78) & $6.08(3.58,11.52)$ & $\dot{0.0001}$ & 0.0002 & $\begin{array}{l}0.6296(0.6084, \\
0.6509)\end{array}$ \\
\hline DM & 2379 & 803 & 1576 & 58.89 & 62.16 & $30.52(66.10)$ & $8.9(4.61,21.39)$ & $\begin{array}{l}<.0001 \\
0.000\end{array}$ & $\begin{array}{l}< \\
0.0001\end{array}$ & $\begin{array}{l}0.7268(0.7091 \\
0.7446)\end{array}$ \\
\hline \multicolumn{11}{|c|}{ Liver cancer } \\
\hline NM & 3446 & 554 & 2892 & 56.82 & 55.36 & $14.13(41.30)$ & $4.915(3.04,8.70)$ & $\dot{0.0001}$ & - & $\begin{array}{l}0.5675(0.5467 \\
0.5883)\end{array}$ \\
\hline LNM & 450 & 82 & 368 & 56.29 & 55.76 & 25.93(59.54) & $6.535(3.55,15.63)$ & $\dot{0} 0001$ & $\dot{0} 0001$ & $\begin{array}{l}0.6537(0.6216 \\
0.6859)\end{array}$ \\
\hline DM & 451 & 75 & 376 & 56.88 & 54.55 & $30.88(69.07)$ & $6.93(3.85,18.92)$ & $\begin{array}{l}<.0001 \\
0.000\end{array}$ & $\begin{array}{l}<.0001 \\
0.000\end{array}$ & $\begin{array}{l}0.6841(0.6533, \\
0.7149)\end{array}$ \\
\hline \multicolumn{11}{|c|}{ Testicular cancer } \\
\hline NM & 10 & 0 & 10 & 0.00 & 64.20 & 44.38 (119.34) & $5.65(3.76,10.57)$ & 0.2684 & - & $\begin{array}{l}0.6377(0.4697 \\
0.8058)\end{array}$ \\
\hline LNM & 6 & 0 & 6 & 0 & 37.67 & $22.68(18.23)$ & $22.255(12.43,23.71)$ & 0.0065 & 0.1471 & $0.8479(0.6379,1.0$ \\
\hline \multicolumn{11}{|c|}{ Cervical cancer } \\
\hline NM & 1889 & 1889 & 0 & 51.27 & 0 & $9.84(16.05)$ & $6.3(3.9,10.96)$ & $\begin{array}{l}< \\
0.0001\end{array}$ & - & $\begin{array}{l}0.6403(0.6186, \\
0.6621)\end{array}$ \\
\hline LNM & 188 & 188 & 0 & 50.85 & 0 & $25.32(55.11)$ & $9.095(5.52,19.65)$ & $\dot{0} 0001$ & $\begin{array}{l}< \\
0.0001\end{array}$ & $0.744(0.7016,0.78$ \\
\hline DM & 92 & 92 & 0 & 52.13 & 0 & $18.29(40.10)$ & $7.94(4.45,15.53)$ & $\begin{array}{l}<.0001 \\
0.000\end{array}$ & 0.0252 & $\begin{array}{l}0.7168(0.6628, \\
0.7708)\end{array}$ \\
\hline
\end{tabular}

SD standard deviation,LNM lymph node metastasis, DM distant metastasis.

The ROC were constructed for NM, LNM or DM vs healthy control 


\begin{tabular}{|c|c|c|c|c|c|c|c|c|c|c|}
\hline & & Count & & Age & & & & $p$ value & & ROC \\
\hline \multicolumn{11}{|c|}{ Laryngocarcinoma } \\
\hline NM & 72 & 4 & 68 & 60.75 & 64.23 & 7.39(6.30) & $5.61(3.62,8.68)$ & 0.0393 & - & $0.6079(0.5451,0.6)$ \\
\hline LNM & 286 & 277 & 64.33 & 62.76 & 63.38 & $7.19(8.40)$ & $5.13(2.87,8.95)$ & 0.0507 & 0.3599 & $0.5589(0.5199,0.5$ \\
\hline DM & 31 & 0 & 31 & 0.00 & 64.35 & 10.77(9.98) & $9(4.43,12.44)$ & 0.3829 & 0.8207 & $0.6781(0.5709,0.7$ \\
\hline \multicolumn{11}{|c|}{$\begin{array}{l}\text { Periampullary } \\
\text { carcinoma }\end{array}$} \\
\hline NM & 56 & 19 & 37 & 57.21 & 56.84 & $29.03(68.28)$ & $6.31(3.76,27.07)$ & 0.0001 & - & $\begin{array}{l}0.6643(0.5792 \\
0.7493)\end{array}$ \\
\hline LNM & 8 & 5 & 3 & 65.20 & 54.00 & $51.41(59.82)$ & $30.35(8.39,58.23)$ & 0.0009 & 0.2341 & $0.871(0.7417,1)$ \\
\hline DM & 19 & 7 & 12 & 52.57 & 62.08 & 101.91(136.96) & $\begin{array}{l}19.56 \\
(11.37,110.625)\end{array}$ & $\begin{array}{l}<.0001 \\
0.00\end{array}$ & 0.0187 & $\begin{array}{l}0.8504(0.7223 \\
0.9784)\end{array}$ \\
\hline \multicolumn{11}{|c|}{ Thyroid cancer } \\
\hline NM & 2771 & 2134 & 637 & 46.2 & 46.24 & $6.83(11.03)$ & $4.78(2.94,8.01)$ & 0.0003 & - & $\begin{array}{l}0.5434(0.5215 \\
0.5653)\end{array}$ \\
\hline LNM & 648 & 449 & 199 & 42.98 & 42.08 & $6.64(8.95)$ & $4.795(2.83,8.25)$ & 0.0073 & $\overrightarrow{0.9999}$ & $0.5451(0.516,0.57$ \\
\hline DM & 58 & 42 & 16 & 50.24 & 46.75 & $8.45(11.22)$ & $5.09(3.17,9.25)$ & 0.194 & $\begin{array}{l}> \\
0.9999\end{array}$ & $0.5711(0.4932,0.6$ \\
\hline \multicolumn{11}{|c|}{ Colorectal cancer } \\
\hline NM & 2990 & 1109 & 1881 & 60.52 & 61.46 & $14.64(34.34)$ & $7.00(4.27,12.58)$ & $<.0001$ & - & $0.6775(0.658,0.69$ \\
\hline LNM & 499 & 206 & 293 & 59.37 & 59.93 & $32.71(63.74)$ & $10.9(6.03,25.46)$ & $<.0001$ & $<.0001$ & $\begin{array}{l}0.7917(0.7665 \\
0.8169)\end{array}$ \\
\hline DM & 997 & 362 & 635 & 58.01 & 60.91 & 58.95 (94.39) & $16.84(6.99,63.17)$ & $<.0001$ & $<.0001$ & $\begin{array}{l}0.8299(0.8117 \\
0.8481)\end{array}$ \\
\hline \multicolumn{11}{|c|}{ Lymphoma } \\
\hline NM & 27 & 13 & 14 & 53.85 & 56.43 & $6.014(4.55)$ & $4.33(2.76,7.03)$ & $\begin{array}{l}> \\
0.9999\end{array}$ & - & $\begin{array}{l}0.5304(0.4207 \\
0.6401)\end{array}$ \\
\hline LNM & 2 & 2 & - & 47.5 & 0.00 & $40.26(45.49)$ & $40.255(24.17,56.33$ & 0.1572 & 0.1997 & $0.8957(0.7503,1.0$ \\
\hline DM & 4 & 3 & 1 & 16.00 & 52.67 & $12.76(7.41)$ & 13.855(7.78,18.83) & 0.0816 & 0.1607 & $0.8193(0.6181,1.0$ \\
\hline \multicolumn{11}{|c|}{ Ovarian cancer } \\
\hline NM & 603 & 604 & 0 & 51.34 & 0.00 & 21.11(49.22) & $7.56(3.83,16.36)$ & $<.0001$ & - & $0.6773(0.6488,0.7($ \\
\hline LNM & 96 & 96 & 0 & 53.21 & 0 & $20.72(32.06)$ & $9.39(6.00,19.75)$ & $\begin{array}{l}<.0001 \\
0.00\end{array}$ & 0.3988 & $0.7852(0.7374,0.8$ \\
\hline DM & 102 & 102 & 0 & 54.32 & 0.00 & $34.86(63.61)$ & $10.95(4.93,28.99)$ & $<.0001$ & 0.053 & $\begin{array}{l}0.7324(0.6966, \\
0.7682)\end{array}$ \\
\hline \multicolumn{11}{|c|}{ Bladder cancer } \\
\hline NM & 466 & 79 & 387 & 63.90 & 66.56 & 8.78(21.95) & $4.7(3,7.98)$ & 0.036 & - & $05487(0.5166,0.58$ \\
\hline LNM & 11 & 1 & 10 & 64.00 & 70.70 & $32.20(84.81)$ & $5.1(3.6,8.03)$ & 0.245 & 0.7051 & $0.6051(0.4718,0.7 \AA$ \\
\hline DM & 34 & 5 & 29 & 61.00 & 68.10 & $34.51(67.81)$ & $9.88(5.25,20.10)$ & $\begin{array}{l}<.0001 \\
0.0\end{array}$ & $\begin{array}{l}< \\
0.0001\end{array}$ & $0.7453(0.6469,0.8<$ \\
\hline
\end{tabular}

Skin cancer

SD standard deviation,LNM lymph node metastasis, DM distant metastasis.

The ROC were constructed for NM, LNM or DM vs healthy control 


\begin{tabular}{|c|c|c|c|c|c|c|c|c|c|c|}
\hline & & Count & & Age & & & & $\mathrm{p}$ value & & ROC \\
\hline NM & 176 & 87 & 89 & 65.69 & 63.5 & $6.14(4.60)$ & $5.01(3.10,7.91)$ & 0.0669 & - & $\begin{array}{l}0.5544(0.5091 \\
0.5997)\end{array}$ \\
\hline LNM & 14 & 5 & 9 & 51.00 & 52.89 & $7.12(6.00)$ & $5.215(3.89,7.21)$ & 0.5778 & $\overrightarrow{0.9999}$ & $\begin{array}{l}0.6021(0.4843 \\
0.7198)\end{array}$ \\
\hline DM & 2 & 1 & 1 & 84.00 & 52.00 & $5.25(1.85)$ & $5.25(4.60,5.91)$ & $\overrightarrow{0.9999}$ & $\overrightarrow{0.9999}$ & $0.581(0.4195,0.74$ \\
\hline \multicolumn{11}{|c|}{ Prostatic cancer } \\
\hline NM & 499 & 0 & 499 & 0.00 & 72.90 & 8.30 (14.17) & $5.02(3.17,8.64)$ & $\hat{0} .0001$ & - & $\begin{array}{l}0.5677(0.5363 \\
0.5991)\end{array}$ \\
\hline LNM & 27 & 0 & 27 & 0.00 & 72.63 & 7.77 (5.078) & $6.77(4.06,8.8)$ & 0.014 & 0.3661 & $0.6626(0.573,0.75$ \\
\hline DM & 201 & 0 & 201 & 0.00 & 73.29 & 11.887(31.77) & $5.45(3.03,10.51)$ & 0.0001 & 0.9397 & $0.5913(0.547,0.63$ \\
\hline \multicolumn{11}{|c|}{ Breast cancer } \\
\hline NM & 2843 & 2843 & 0 & 50.73 & 0 & $8.13(8.92)$ & $5.94(3.59,9.80)$ & $\hat{0}_{0.0001}^{<}$ & - & $0.614(0.593,0.634$ \\
\hline LNM & 454 & 454 & 0 & 50.87 & 0.00 & $10.39(14.40)$ & $6.775(4.42,11.99)$ & $\stackrel{<}{0.0001}$ & $\dot{<} 0001$ & $\begin{array}{l}0.6788(0.6489 \\
0.7087)\end{array}$ \\
\hline DM & 527 & 527 & 0 & 52.15 & 0.00 & $16.68(35.00)$ & $7.87(4.44,14.32)$ & $\hat{0}_{0.0001}$ & $\hat{0}_{0.0001}$ & $\begin{array}{l}0.6979(0.6692 \\
0.7267)\end{array}$ \\
\hline \multicolumn{11}{|c|}{$\begin{array}{l}\text { Neuroendocrine } \\
\text { carcinoma }\end{array}$} \\
\hline NM & 24 & 14 & 10 & 49.36 & 59.30 & $12.25(23.39)$ & 7.175(3.87,9.29) & 0.0099 & - & $0.653(0.5484,0.75$ \\
\hline LNM & 1 & 0 & 1 & 0.00 & 50.00 & 10.02() & $10.02(10.02,10.02)$ & & - & $\begin{array}{l}0.8682(0.8458 \\
0.8905)\end{array}$ \\
\hline \multicolumn{11}{|c|}{ Renal Cell Cancers } \\
\hline NM & 791 & 306 & 485 & 58.02 & 58.08 & $12.90(37.39)$ & $5.4(3.18,9.9)$ & $\begin{array}{l}< \\
0.0001\end{array}$ & - & $0.5939(0.5668,0.6$ \\
\hline LNM & 29 & 5 & 24 & 59.40 & 57.71 & $9.48(10.26)$ & $4.91(3.01,10.17)$ & 0.2791 & $\overrightarrow{0.9999}$ & $\begin{array}{l}0.5899(0.4721 \\
0.7078)\end{array}$ \\
\hline DM & 82 & 22 & 60 & 54.27 & 61.25 & $14.39(52.16)$ & $5.21(2.81,10.70)$ & 0.079 & $\overrightarrow{0} .9999$ & $0.572(0.5019,0.64$ \\
\hline \multicolumn{11}{|c|}{ Esophagus cancer } \\
\hline NM & 1034 & 239 & 795 & 64.01 & 63.7 & $9.561(30.12)$ & $4.96(3.08,8.06)$ & $\begin{array}{l}< \\
0.0001\end{array}$ & - & $\begin{array}{l}0.5596(0.5339 \\
0.5854)\end{array}$ \\
\hline LNM & 169 & 19 & 150 & 64.95 & 64.87 & $14.846(52.62)$ & $6.01(3.35,10.71)$ & $\hat{0}_{0.0001}$ & 0.0175 & $\begin{array}{l}0.6232(0.5771 \\
0.6692)\end{array}$ \\
\hline DM & 125 & 14 & 111 & 63.5 & 63.77 & $17.17(48.23)$ & $6.18(3.39,10.97)$ & 0.0001 & 0.1068 & $0.6102(0.554,0.66$ \\
\hline \multicolumn{11}{|c|}{$\begin{array}{l}\text { Fallopian Tube } \\
\text { Cancer }\end{array}$} \\
\hline NM & 6 & 6 & 0 & 67.67 & 0 & $4.91(3.89)$ & $3.695(3.41,5.28)$ & $\vec{D} .9999$ & - & $\begin{array}{l}0.5284(0.3134 \\
0.7435)\end{array}$ \\
\hline DM & 4 & 4 & 0 & 53 & 0 & $10.17(4.11)$ & $9.405(7.49,12.08)$ & 0.0487 & - & $\begin{array}{l}0.8257(0.7163 \\
0.9352)\end{array}$ \\
\hline \multicolumn{11}{|c|}{ Ureter cancer } \\
\hline NM & 9 & 8 & 1 & 53 & 69 & $28.09(45.31)$ & $7.66(4.47,8.5)$ & $\overrightarrow{>} .9999$ & - & $0.672(0.4915,0.85$ \\
\hline LNM & 1 & 0 & 1 & 0 & 82 & 33.15() & $33.15(33.15,33.15)$ & 0.0487 & 0.7273 & $0.8705(0.69,1.051$ \\
\hline DM & 2 & 1 & 1 & 52 & 62 & $25.34(25.77$ & $25.34(16.23,34.45)$ & & - & $0.9989(0.9966,1.0$ \\
\hline \multicolumn{11}{|c|}{ SD standard deviation,LNM lymph node metastasis, DM distant metastasis. } \\
\hline & & $1 \mathrm{~N}$ & & & & & & & & \\
\hline
\end{tabular}




\begin{tabular}{|c|c|c|c|c|c|c|c|c|c|c|}
\hline & & Count & & Age & & & & $\mathrm{p}$ value & & ROC \\
\hline \multicolumn{11}{|c|}{$\begin{array}{l}\text { Craniofacial } \\
\text { malignant tumour }\end{array}$} \\
\hline NM & 262 & 82 & 180 & 57.79 & 59.17 & $9.95(23.29)$ & $6.25(3.65,9.29)$ & $\stackrel{<}{0.0001}$ & - & $\begin{array}{l}0.6204(0.5821 \\
0.6586)\end{array}$ \\
\hline LNM & 119 & 28 & 91 & 56.79 & 59.16 & 10.77(10.98) & $7.81(4.32,12.05)$ & $<.0001$ & 0.0244 & $\begin{array}{l}0.5933(0.4295 \\
0.7571)\end{array}$ \\
\hline DM & 14 & 1 & 13 & 77 & 60.17 & $7.35(6.19)$ & $6.3(3.3,8.10)$ & 0.6664 & $\vec{D} .9999$ & $0.6936(0.642,0.74$ \\
\hline \multicolumn{11}{|c|}{ Vulvar cancer } \\
\hline NM & 35 & 35 & 59 & 0 & 0 & $9.15(7.11)$ & $7.99(4.45,12.25)$ & 0.0007 & - & $\begin{array}{l}0.6825(0.5837 \\
0.7813)\end{array}$ \\
\hline LNM & 10 & 10 & 53.4 & 0 & 0 & $3.69(1.10)$ & $3.49(3.1,4.47)$ & $\vec{D} .9999$ & 0.01 & $0.5855(0.499,0.67$ \\
\hline DM & 7 & 7 & 61.14 & 0 & 0 & $7.08(4.32)$ & $7.31(4.08,10.63)$ & 0.824 & $\vec{D} .9999$ & $\begin{array}{l}0.6196(0.3764 \\
0.8629)\end{array}$ \\
\hline \multicolumn{11}{|c|}{ Gastric cancer } \\
\hline NM & 1315 & 410 & 905 & 58.29 & 61.05 & $20.72(51.68)$ & $6.4(3.67,12.53)$ & $\stackrel{<}{0.0001}$ & - & $0.647(0.6242,0.66$ \\
\hline LNM & 460 & 139 & 321 & 55.78 & 62.45 & $34.65(74.86)$ & $8.975(5.52,19.34)$ & <. 0001 & $<.0001$ & $\begin{array}{l}0.7623(0.7355 \\
0.7891)\end{array}$ \\
\hline DM & 240 & 86 & 154 & 54.92 & 61.22 & $55.39(100.78)$ & $10.40(4.33,43.78)$ & $\hat{L}_{0.0001}$ & $\hat{L}_{0.0001}^{<}$ & $\begin{array}{l}0.7359(0.6955 \\
0.7764)\end{array}$ \\
\hline \multicolumn{11}{|c|}{ Thymic cancer } \\
\hline NM & 12 & 3 & 9 & 45.67 & 60.22 & $6.77(6.81)$ & $3.51(2.74,7.57)$ & $\overrightarrow{>} .9999$ & - & $0.5046(0.3393,0.6$ \\
\hline LNM & 1 & 1 & 0 & 48 & 0 & 28.13() & $28.13(28.13,28.13)$ & $\overrightarrow{>} .9999$ & 0.9451 & $0.5097(0.156,0.86$ \\
\hline DM & 3 & 1 & 2 & 46 & 59.5 & $5.64(4.74)$ & $4.41(3.02,7.64)$ & & - & $0.9977(0.9946,1.0$ \\
\hline \multicolumn{11}{|c|}{ Pancreatic cancer } \\
\hline NM & 335 & 149 & 186 & 60.28 & 61.21 & 75.64(108.64) & $22.71(6.97,95.74)$ & $\stackrel{<}{0.0001}$ & - & $\begin{array}{l}0.8247(0.7947 \\
0.8547)\end{array}$ \\
\hline LNM & 62 & 18 & 44 & 64.06 & 59.66 & $79.73(116.23)$ & $30.19(7.85,100.37)$ & $<.0001$ & $\overrightarrow{>} .9999$ & $0.8587(0.799,0.91$ \\
\hline DM & 253 & 93 & 160 & 58.94 & 60.28 & 122.53(131.12) & $57.66(17.42,189.06)$ & $\hat{0}_{0.0001}^{<}$ & $\iota_{0.0001}^{<}$ & $\begin{array}{l}0.9113(0.8863 \\
0.9362)\end{array}$ \\
\hline \multicolumn{11}{|c|}{ Vaginal cancer } \\
\hline NM & 14 & 14 & 0 & 52.21 & 0 & 27.57 (58.09) & $8.105(5.00,11.54)$ & 0.0055 & - & $\begin{array}{l}0.7422(0.6302 \\
0.8543)\end{array}$ \\
\hline LNM & 3 & 3 & 0 & 51.67 & 0 & 8.02(3.93) & $6.08(5.76,9.31)$ & 0.4835 & $\vec{D} .9999$ & $\begin{array}{l}0.7354(0.5798 \\
0.8911)\end{array}$ \\
\hline DM & 7 & 7 & 0 & 54 & 0 & $14.05(9.51)$ & $14.33(6.19,19.55)$ & 0.015 & $\overrightarrow{0} .9999$ & $\begin{array}{l}0.8075(0.6508 \\
0.9641)\end{array}$ \\
\hline \multicolumn{11}{|c|}{ Penile cancer } \\
\hline NM & 48 & 0 & 48 & 0 & 56.56 & $5.15(4.10)$ & $4.04(2.49,7.04)$ & $\overrightarrow{0.9999}$ & - & $\begin{array}{l}0.5267(0.4416 \\
0.6118)\end{array}$ \\
\hline LNM & 9 & 0 & 9 & 0 & 55.22 & $4.91(4.03)$ & $3.65(2.18,7.1)$ & $\overrightarrow{>} .9999$ & $\overrightarrow{>} .9999$ & $0.547(0.3552,0.73$ \\
\hline DM & 8 & 0 & 8 & 0 & 56.75 & $6.73(6.63)$ & $3.91(2.80,7.61)$ & $\overrightarrow{0} .9999$ & $\vec{l} .9999$ & $0.5056(0.295,0.71$ \\
\hline
\end{tabular}

Endometrial cancer

SD standard deviation,LNM lymph node metastasis, DM distant metastasis.

The ROC were constructed for NM, LNM or DM vs healthy control 


\begin{tabular}{|c|c|c|c|c|c|c|c|c|c|c|}
\hline & & Coun & & Age & & & & $\mathrm{p}$ value & & ROC \\
\hline NM & 398 & 398 & 0 & 55.49 & 0 & 10.59 (16.72) & $6.145(3.76,10.54)$ & $\begin{array}{l}< \\
0.0001\end{array}$ & - & $0.6371(0.6043,0.6$ \\
\hline LNM & 27 & 27 & 0 & 57.78 & 0 & $24.65(23.68)$ & $15.51(10.77,32.66)$ & $\begin{array}{l}< \\
0.0001\end{array}$ & $\begin{array}{l}< \\
0.0001\end{array}$ & $\begin{array}{l}0.8868(0.8217 \\
0.9518)\end{array}$ \\
\hline DM & 13 & 13 & 0 & 55.38 & 0 & $18.76(20.35)$ & $13.41(3.23,19.37)$ & 0.0075 & 0.2417 & $\begin{array}{l}0.7282(0.5379 \\
0.9185)\end{array}$ \\
\hline
\end{tabular}

SD standard deviation,LNM lymph node metastasis, DM distant metastasis.

The ROC were constructed for NM, LNM or DM vs healthy control

We constructed ROC curves for the serum CA242 values of the patients with each type of tumor versus that of healthy controls. The AUC value positively correlated with metastasis in most tumors. In patients with cholangiocarcinoma with LNM or pancreatic cancer with DM, the AUC of CA242 was greater than 0.9 , indicating that CA242 has a high diagnostic value. In 6 diseases including cholangiocarcinoma, gallbladder cancer, periampullary carcinoma, colorectal cancer, gastric cancer, and pancreatic cancer, when LNM or DM appeared, the AUC values were all significantly higher than 0.7, and DM produced a larger increase than LNM (Table 2).

\section{CA242 in different clinical stages}

We analyzed the serum CA242 values of 11 types of tumors in 2,500 patients who had certain clinical staging information.

We found that the mean and median levels of serum CA242 in most tumor patients increased with stage progression and were almost all higher than those in healthy controls. The median CA242 level in stage IV gastric cancer was $22.87(7.33,84.00)$, and that in stage IV colorectal cancer was 20.48 (7.74, 61.255$)$. Both of these values were approximately five times higher than the value for the healthy controls $[4.32(2.46,7.26)]$. The means of some tumors in stage IV, including lung cancer $(31.65 \pm 67.96)$, ovarian cancer $(34.37 \pm 69.27)$ and cervical cancer $(26.04 \pm 47.08)$, were approximately $5-6$ times higher than the mean of the healthy controls $(5.58 \pm 4.56)$, but the corresponding medians were only approximately two times higher. However, the medians in ovarian cancer and lung cancer did not change significantly between stages II and III. No significant differences were observed in the following diseases: nasopharyngeal carcinoma in stage I or II, laryngeal cancer in stage I or II, bladder cancer, renal cancer, esophageal cancer, and endometrial cancer (Table 3 and Fig. 3). 
Table 3

CA242 in different clinical stages

\begin{tabular}{|c|c|c|c|c|c|c|c|c|c|c|}
\hline & & Count & & Age & & & & $P$ value & & ROC \\
\hline & Case & Female & Male & Female & Male & mean(SD) & median(25\%,75\&) & $\begin{array}{l}\text { control } \\
\text { vs }\end{array}$ & Ivs & $\begin{array}{l}\text { AUC(95\% } \\
\text { confidence } \\
\text { interval) }\end{array}$ \\
\hline Colorectal cancer & 2205 & 823 & 1382 & 59.07 & 60.13 & $28.19(45.2)$ & $11.0725(5.66,10.95)$ & - & - & - \\
\hline 1 & 517 & 200 & 317 & 59.82 & 60.74 & $9.97(11.2)$ & $6.95(4.68,10.95)$ & $<.0001$ & - & $\begin{array}{l}0.684(0.658 \\
0.7099)\end{array}$ \\
\hline II & 795 & 280 & 515 & 61.44 & 62.10 & 11.8(12.77) & $8.04(4.98,13.22 b)$ & $<.0001$ & $<.0001$ & $\begin{array}{l}0.7394(0.7171, \\
0.7617)\end{array}$ \\
\hline III & 742 & 285 & 457 & 58.48 & 59.84 & $24.32(54.21)$ & $8.82(5.24,17.54 b)$ & $<.0001$ & $\begin{array}{l}<.0001 \\
0.00\end{array}$ & $\begin{array}{l}0.7431(0.7191 \\
0.7671)\end{array}$ \\
\hline IV & 151 & 58 & 93 & 56.53 & 57.84 & $66.67(102.62)$ & $20.48(7.74,61.255 b)$ & $<.0001$ & $\begin{array}{l}<.0001 \\
0.00\end{array}$ & $\begin{array}{l}0.8612(0.8235 \\
0.899)\end{array}$ \\
\hline $\begin{array}{l}\text { Nasopharyngeal } \\
\text { carcinoma }\end{array}$ & 215 & 57 & 158 & 47.40 & 50.84 & $11.1525(13.6875)$ & $6.7725(4.285,13.9825)$ & - & - & - \\
\hline 1 & 3 & 2 & 1 & 43.00 & 48.00 & $8.56(8.67)$ & $4.88(3.61,11.67)$ & > 0.9999 & - & $\begin{array}{l}0.6065(0.08769, \\
1.125)\end{array}$ \\
\hline II & 13 & 3 & 10 & 47.67 & 53.90 & $11.15(8.7)$ & $7.89(5.74,16.02)$ & > 0.9999 & > 0.9999 & $\begin{array}{l}0.6028(0.3338 \\
0.8719)\end{array}$ \\
\hline III & 91 & 26 & 65 & 49.27 & 50.42 & $10.91(13.63)$ & $6.39(3.47,12.82)$ & $<.0001$ & D.9999 & $\begin{array}{l}0.6537(0.5929 \\
0.7145)\end{array}$ \\
\hline IV & 108 & 26 & 82 & 49.65 & 51.04 & 13.99(23.75) & 7.93(4.32,15.42) & $<.0001$ & > 0.9999 & $\begin{array}{l}0.7098(0.6529, \\
0.7668)\end{array}$ \\
\hline Lung cancer & 2731 & 933 & 1798 & 60.00 & 61.91 & $17.59(40.6675)$ & $6.4275(3.93,12.81)$ & - & - & - \\
\hline 1 & 1522 & 531 & 991 & 58.76 & 61.05 & 11.02(21.6) & $6.1(3.64,10.83)$ & $\begin{array}{l}< \\
0.0001\end{array}$ & - & $\begin{array}{l}0.6301(0.6073 \\
0.6528)\end{array}$ \\
\hline II & 28 & 4 & 24 & 63.25 & 60.75 & $15.46(33.41)$ & $4.67(3.34,9.01)$ & 0.5444 & > 0.9999 & $\begin{array}{l}0.5808(0.4727 \\
0.6889)\end{array}$ \\
\hline III & 57 & 16 & 41 & 59.63 & 64.29 & 12.23(39.7) & $5.5(3.84,8.56)$ & 0.1466 & $\begin{array}{l}> \\
0.9999\end{array}$ & $\begin{array}{l}0.6009(0.5284 \\
0.6733)\end{array}$ \\
\hline IV & 1124 & 382 & 742 & 58.36 & 61.53 & $31.65(67.96)$ & $9.44(4.9,22.84)$ & $<.0001$ & $<.0001$ & $\begin{array}{l}0.8101(0.7941 \\
0.8262)\end{array}$ \\
\hline Cervical cancer & 999 & 999 & 0 & 50.57 & 0.00 & 14.8775(25.36) & $6.9275(4.645,13.7825)$ & - & - & - \\
\hline I & 519 & 519 & 0 & 48.70 & 0 & $10.06(21.11)$ & $6.32(4.09,10.76)$ & $<.0001$ & - & $\begin{array}{l}0.652(0.6226 \\
0.6813)\end{array}$ \\
\hline II & 434 & 434 & 0 & 52.58 & 0 & 11.37(15.01) & $7.4(4.44,12.63)$ & $\begin{array}{l}< \\
0.0001\end{array}$ & 0.0762 & $\begin{array}{l}0.6875(0.6569 \\
0.7182)\end{array}$ \\
\hline III & 31 & 31 & 0 & 52.74 & 0 & $12.04(18.24)$ & $5.88(3.93,10.86)$ & 0.0606 & > 0.9999 & $\begin{array}{l}0.6215(0.5142, \\
0.7289)\end{array}$ \\
\hline IV & 15 & 15 & 0 & 48.27 & 0 & 26.04(47.08) & $8.11(6.12,20.88)$ & $<.0001$ & 0.0431 & $\begin{array}{l}0.8324(0.7498 \\
0.9149)\end{array}$ \\
\hline Laryngocarcinoma & 268 & 9 & 259 & 59.77 & 63.39 & $7.0975(6.435)$ & $5.0025(3.0275,9.0025)$ & - & - & - \\
\hline I & 131 & 3 & 128 & 58.33 & 61.24 & $6.12(4.61)$ & $5.01(2.7,8.04)$ & 0.3158 & - & $\begin{array}{l}0.5475(0.4938 \\
0.6012)\end{array}$ \\
\hline II & 55 & 4 & 51 & 66.75 & 63.73 & $5.74(4.4)$ & $4.05(2.37,8.63)$ & $\begin{array}{l}> \\
0.9999\end{array}$ & $\begin{array}{l}> \\
0.9999\end{array}$ & $\begin{array}{l}0.5038(0.4182 \\
0.5894)\end{array}$ \\
\hline III & 44 & 1 & 43 & 40.00 & 64.84 & $6.12(4.23)$ & $5.06(2.9,9.15)$ & > 0.9999 & > 0.9999 & $\begin{array}{l}0.5468(0.4536 \\
0.64)\end{array}$ \\
\hline IV & 38 & 1 & 37 & 74.00 & 63.76 & $10.41(12.5)$ & $5.89(4.14,10.19)$ & 0.0168 & 0.6204 & $\begin{array}{l}0.638(0.5503, \\
0.7256)\end{array}$ \\
\hline Ovarian cancer & 624 & 624 & 0 & 51.26 & 0.00 & 19.525(31.8825) & $8.955(5.1675,18.6225)$ & - & - & - \\
\hline
\end{tabular}




\begin{tabular}{|c|c|c|c|c|c|c|c|c|c|c|}
\hline & & Count & & Age & & & & $P$ value & & ROC \\
\hline 1 & 127 & 127 & 0 & 48.08 & 0 & 11.55(10.95) & 7.62(4.87,15.39) & $\begin{array}{l}<.0001 \\
0.00\end{array}$ & - & $\begin{array}{l}0.7377(0.6933 \\
0.7821)\end{array}$ \\
\hline II & 78 & 78 & 0 & 51.50 & 0 & 13.15(12.35) & $8.5(5.92,14.05)$ & $\begin{array}{l}< \\
0.0001\end{array}$ & $\overrightarrow{0.9999}$ & $\begin{array}{l}0.7676(0.7151 \\
0.8201)\end{array}$ \\
\hline III & 334 & 334 & 0 & 53.42 & 0 & 19.03(34.96) & $8.73(4.91,16.74)$ & $\begin{array}{l}<.0001 \\
0\end{array}$ & $\overrightarrow{0.9999}$ & $\begin{array}{l}0.7326(0.7001 \\
0.7652)\end{array}$ \\
\hline IV & 85 & 85 & 0 & 52.04 & 0 & $34.37(69.27)$ & $10.97(4.97,28.31)$ & $\begin{array}{l}<.0001 \\
0.00\end{array}$ & 0.414 & $\begin{array}{l}0.7709(0.7134 \\
0.8284)\end{array}$ \\
\hline Bladder cancer & 66 & 10 & 56 & 44.04 & 69.26 & $12.1525(16.62)$ & $6.02(3.5475,10.9575)$ & - & - & - \\
\hline I & 20 & 2 & 18 & 48.50 & 65.72 & $5.23(4.37)$ & $4.1(3.6,4.67)$ & > 0.9999 & - & $\begin{array}{l}0.502(0.4086 \\
0.5953)\end{array}$ \\
\hline II & 14 & 0 & 14 & 0 & 70.80 & $7.32(5.44)$ & $5.62(3.84,8.26)$ & 0.1614 & 0.7376 & $\begin{array}{l}0.6547(0.5372 \\
0.7721)\end{array}$ \\
\hline III & 11 & 2 & 9 & 72.67 & 70.38 & $5.78(4.3)$ & $5(2.72,7.51)$ & $\begin{array}{l}> \\
0.9999\end{array}$ & $\overrightarrow{0.9999}$ & $\begin{array}{l}0.5275(0.3561, \\
0.6989)\end{array}$ \\
\hline IV & 21 & 6 & 15 & 55.00 & 70.13 & $30.28(52.37)$ & $9.36(4.03,23.39)$ & 0.006 & 0.0955 & $\begin{array}{l}0.7015(0.5636 \\
0.8393)\end{array}$ \\
\hline Renal carcinoma & 95 & 31 & 64 & 58.76 & 57.57 & $15.205(23.9775)$ & $4.9275(3.3875,13.455)$ & - & - & - \\
\hline I & 46 & 13 & 33 & 60.33 & 55.09 & $6.4(4.1)$ & $5.65(3.5,8.75)$ & 0.269 & - & $\begin{array}{l}0.5806(0.4967 \\
0.6646)\end{array}$ \\
\hline II & 10 & 5 & 5 & 56.60 & 61.71 & $5.98(6.33)$ & $3.04(1.79,8.35)$ & > 0.9999 & $\overrightarrow{0.9999}$ & $\begin{array}{l}0.5659(0.3485 \\
0.7832)\end{array}$ \\
\hline III & 17 & 5 & 12 & 64.60 & 55.33 & $9.2(7.84)$ & $5.43(4.15,14.99)$ & 0.3551 & $\begin{array}{l}> \\
0.9999\end{array}$ & $\begin{array}{l}0.6199(0.4626 \\
0.7772)\end{array}$ \\
\hline IV & 22 & 8 & 14 & 53.50 & 58.14 & $39.24(77.64)$ & $5.59(4.11,21.73)$ & 0.014 & 0.8486 & $\begin{array}{l}0.6818(0.5704 \\
0.7932)\end{array}$ \\
\hline Esophagus cancer & 547 & 92 & 455 & 63.46 & 62.13 & $9.7475(19.805)$ & $5.2525(3.435,8.4225)$ & - & - & - \\
\hline I & 99 & 26 & 73 & 63.46 & 63.05 & $5.78(3.36)$ & $5.2(3.45,7.12)$ & 0.1949 & - & $\begin{array}{l}0.5624(0.5103 \\
0.6144)\end{array}$ \\
\hline II & 241 & 42 & 199 & 61.42 & 62.96 & $6.77(6.32)$ & $5.12(3.11,7.85)$ & 0.0094 & $\overrightarrow{0.9999}$ & $\begin{array}{l}0.5643(0.5249 \\
0.6037)\end{array}$ \\
\hline III & 187 & 21 & 166 & 62.29 & 63.04 & $16.38(54.94)$ & $5.48(3.34,10.56)$ & $\begin{array}{l}<.0001 \\
0.00\end{array}$ & $\overrightarrow{0.9999}$ & $\begin{array}{l}0.6105(0.5655 \\
0.6555)\end{array}$ \\
\hline IV & 20 & 3 & 17 & 66.67 & 59.47 & $10.06(14.6)$ & $5.21(3.84,8.16)$ & 0.6519 & $\overrightarrow{0.9999}$ & $\begin{array}{l}0.5892(0.4611, \\
0.7174)\end{array}$ \\
\hline Gastric cancer & 1031 & 316 & 715 & 55.74 & 60.52 & $30.6025(48.2)$ & $11.2075(5.15,10.815)$ & - & - & - \\
\hline I & 242 & 65 & 177 & 58.23 & 59.83 & $7.92(6.33)$ & $6.25(3.76,9.98)$ & $\begin{array}{l}<.0001 \\
0.000\end{array}$ & - & $\begin{array}{l}0.6295(0.5901, \\
0.669)\end{array}$ \\
\hline II & 191 & 67 & 124 & 53.82 & 60.68 & $11.27(14.55)$ & $6.97(4.48,11.65)$ & $<.0001$ & 0.4399 & $\begin{array}{l}0.6801(0.6391, \\
0.7211)\end{array}$ \\
\hline III & 496 & 139 & 357 & 56.01 & 61.06 & $33.25(71.93)$ & $8.74(5.03,20.64 b)$ & $\begin{array}{l}<.0001 \\
0.00\end{array}$ & $\begin{array}{l}< \\
0.0001\end{array}$ & $\begin{array}{l}0.7489(0.722 \\
0.7758)\end{array}$ \\
\hline IV & 102 & 45 & 57 & 54.89 & 60.51 & 69.97(99.99) & $22.87(7.33,84.00 \mathrm{~b})$ & $\begin{array}{l}<.0001 \\
0.00\end{array}$ & $\begin{array}{l}<.0001 \\
0\end{array}$ & $\begin{array}{l}0.8508(0.8073 \\
0.8942)\end{array}$ \\
\hline $\begin{array}{l}\text { Endometrial } \\
\text { cancer }\end{array}$ & 104 & 104 & 0 & 56 & 0 & $11.8275(9.5425)$ & $9.3(6.2725,14.5)$ & - & - & - \\
\hline I & 85 & 85 & 0 & 56.57 & 0 & $8.12(6.85)$ & $5.67(3.73,10.19)$ & 0.0008 & - & $\begin{array}{l}0.6228(0.5594 \\
0.6863)\end{array}$ \\
\hline II & 4 & 4 & 0 & 59.50 & 0 & $17.53(14.04)$ & $13.21(8.27,22.46)$ & 0.0402 & 0.3852 & $\begin{array}{l}0.8703(0.7426 \\
0.998)\end{array}$ \\
\hline III & 13 & 13 & 0 & 57.77 & 0 & 13.8(11.31) & $10.46(7.34,15.38)$ & 0.0028 & 0.2008 & $\begin{array}{l}0.7705(0.6031 \\
0.9378)\end{array}$ \\
\hline
\end{tabular}




\begin{tabular}{|c|c|c|c|c|c|c|c|c|c|c|}
\hline & & Count & & Age & & & & $P$ value & & ROC \\
\hline IV & 2 & 2 & 0 & 49.50 & 0 & $7.86(5.97)$ & $7.86(5.75,9.97)$ & $\begin{array}{l}> \\
0.9999\end{array}$ & $\overrightarrow{0} .9999$ & $\begin{array}{l}0.6679(0.321 \\
1.015)\end{array}$ \\
\hline
\end{tabular}

SD standard deviation

The ROC were constructed for stage I, stage II, stage III or stage IV vs healthy control

Then, the ROC of the CA242 level in each tumor stage relative to that in the healthy controls was calculated. The analysis showed that the AUC of CA242 gradually increased with progression in most tumors. Among these tumors, colorectal cancer, gastric cancer, cervical cancer, and lung cancer were particularly remarkable. Especially in stage IV, the AUC values of these four tumors were greater than 0.8, specifically $0.8612(0.8235,0.899)$ for colorectal cancer, 0.8508 (0.8073 0.8942) for gastric cancer, $0.8324(0.7498,0.9149)$ for cervical cancer, and $0.8101(0.7941,0.8262)$ for lung cancer, showing that CA242 was valuable in predicting stage IV disease for these four tumors (Table 3 and Fig. 3).

\section{CA242 in different metastatic sites}

Based on the above results, we noticed that serum CA242 levels exhibited a significant change with DM and had the capacity to predict metastasis in some tumors. Therefore, we wanted to study whether there is a correlation between patient serum CA242 levels and sites of metastasis. Therefore, we divided 3,953 patients with one of five cancers who had defined metastatic site information into group NM and group DM.

We found that the mean and median values of the serum CA242 level in DM patients in most diseases were higher than those in NM patients and healthy controls, but there were no significant differences among the patients with different metastatic sites within each tumor type (Supplemental Table 3). We speculated that this lack of difference was due to the small sample sizes for some metastatic sites and the large variation range of CA242. Therefore, we calculated the median fold change in DM patients relative to NM patients for every tumor type and the log2 (fold change) value of the serum CA242 level for each metastatic site and found the following results: multiple metastases: 1.228 (-0.1189, 3.079), liver metastasis: 1.071 ( $-0.1195,3.059)$, bone metastasis: $0.7286(-0.2386,1.979)$, brain metastasis: $0.6536(0.2648,2.401)$, and lung metastasis: $0.4921(-0.3371,1.676)$. There were significant differences among the metastatic sites other than the brain (Table 4).

Table 4

CA242 in different metastatic sites

\begin{tabular}{|c|c|c|c|c|c|c|c|c|c|c|c|}
\hline & & Count & & Age & & & & $P$ value & & & \\
\hline & Case & Female & Male & Female & Male & Mean(SD) & Median(25\%,75\%) & vs. Liver & vs. Bone & vs. Brain & vs. Multiple \\
\hline Lung & 362 & 112 & 250 & 56.67 & 56.69 & $0.8567(1.889)$ & $0.4921(-0.3371,1.676)$ & 0.0003 & $>0.9999$ & $>0.9999$ & $<0.0001$ \\
\hline Liver & 557 & 167 & 390 & 58.93 & 61.44 & $1.437(2.121)$ & $1.071(-0.1195,3.059)$ & - & 0.0114 & $>0.9999$ & $>0.9999$ \\
\hline Bone & 719 & 244 & 475 & 57.61 & 61.47 & $1.027(1.837)$ & $0.7286(-0.2386,1.979)$ & - & - & $>0.9999$ & 0.0006 \\
\hline Brain & 58 & 23 & 35 & 56.91 & 63.20 & $1.244(1.661)$ & $0.6536(0.2648,2.401)$ & - & - & - & $>0.9999$ \\
\hline Multiple & 604 & 228 & 376 & 57.75 & 60.81 & $1.509(2.167)$ & $1.228(-0.1189,3.079)$ & - & - & - & - \\
\hline
\end{tabular}

\section{Discussion}

Although CA242 has been identified and used in clinical practice for some time, this is the first study to profile the levels of this biomarker in 35 different tumors in a large number of patients. Among the 35 tumor types, the serum CA242 level changes in 23 tumor types had never been reported before. We also found that serum CA242 levels in patients with metastatic tumors might be related to the target organs containing metastases for the first time.

We found that CA242 levels were elevated in a variety of digestive system cancers, including pancreatic cancer, gallbladder cancer, cholangiocarcinoma, colorectal cancer, and gastric cancer, which was consistent with previous reports. The AUC values of the ROC curves for pancreatic cancer, gallbladder carcinoma, and cholangiocarcinoma were all greater than 0.8 , but only pancreatic cancer has been studied in detail[ $[4,10,13,17,22,25-27]$. Previous studies have shown that CA242 alone or in combination with other biomarkers can predict the diagnosis, progression, and prognosis of pancreatic cancer[4, 7, 10, 12$16,23,24]$. Pancreatic cancer has been the leading clinical application for CA242 because of its excellent performance in this cancer. Some small-scale studies have investigated CA242 in gallbladder carcinoma and cholangiocarcinoma[7, 14, 25, 28], but due to the sample size or quality of these studies, the credible AUC values for these two carcinomas are still unknown. Therefore, our findings indicate that CA242 is also suitable for use as a biomarker for cholangiocarcinoma and gallbladder cancer, whose AUCs were $0.8145(0.7737,0.8553)$ and $0.8349(0.8157,0.8542)$, respectively $($ Table 1$)$.

The study of serum CA242 levels in the ampulla of Vater carcinomas, including ampullary carcinoma and duodenal papilla carcinoma, is shown for the first time. A previous study reported that the mean level in the ampulla of Vater carcinoma is $21.1 \pm 26.1$ [29], but the level was $47.87 \pm 93.24$ for the ampulla of Vater carcinoma in our research. Furthermore, the median level was 2.70 times that of healthy controls, and the AUC value reached $0.7268(0.6581,0.7955)$ (Table 1). The discrepancies between the previous study and our study may be due to the sample size in the other study being too small to produce a statistical error or the difference between the ampulla of Vater carcinoma and duodenal papilla carcinoma not being negligible. 
CA242 has been reported to be more sensitive and specific than CA50 and CEA in Dukes' A-D colorectal cancer[22], in addition to its considerable value in predicting recurrence and metastasis in clinical stage II and III colorectal cancer[18]. Moreover, many studies have demonstrated that CA242 works well in predicting the diagnosis, progression, and prognosis of colorectal cancer in combination with other tumor markers[18, 19, 26, 30]. Our results showed that the mean value in colorectal cancer was $26.50 \pm 59.58$ and that the AUC was $0.7241(0.7073,0.7408)$ (Table 1$)$, which was consistent with previous studies[22, 26 , 27].

CA242 levels in patients with gastric cancer differed between stage IV and non-stage IV[20], but alone, CA242 was not a good predictor of LNM[21]. However, in a study by Fangxuan Li et al.[31], the mean value of $29.84 \pm 83.54$ in gastric cancer was not much different from our data, but their AUC for gastric cancer relative to healthy people was 0.809 , which is much higher than the value of $0.6839(0.604,0.8202)$ found in this study (Table 1$)$. We suggest that a possible explanation may be that their sample size is too small and that their mean lower than the control is $3.85 \pm 3.13$. A surprising finding was that the CA242 level in patients with gastric cardia adenocarcinoma was not significantly different from that in gastric cancer patients.

In this study, the mean value in lung cancer was $18.49 \pm 45.97$ (Table 1), but Huaiqian Dou et al.[10] reported that the mean in lung cancer was $9.34 \pm 13.67$. In contrast, Xiaochuan Wang et al. [32] reported that it was $28.39 \pm 51.91$. These differences might be caused by a sampling error, detection kit choice, and instrument differences. However, the results we reported have the largest sample size and therefore higher credibility. The mean value in ovarian cancer was $19.52 \pm 42.27$ (Table 1), which was slightly higher than the previously reported value of $11.40 \pm 16.88[10]$, and the mean in cervical cancer was $11.54 \pm 23.97$, which was very similar to the previously reported value[10]. The levels in nasopharyngeal carcinoma, craniofacial malignant tumor, endometrial cancer, breast cancer, renal cell cancer, liver cancer, esophageal cancer, and prostate cancer patients were significantly higher than those in healthy controls (Table 1), indicating that CA242 has potential clinical value in these diseases. Although mediastinal cancer, testicular cancer, ureteral cancer, and neuroendocrine cancer patients had higher mean and median values than health controls (Table 1), the sample size was too small to identify significant differences.

The CA242 levels in colorectal cancer and gastric cancer patients increased not only with the appearance of LNM or DM but also with advancement from stage I to IV $[18,20-22,26,30]$. The results mentioned above are consistent with the trends in a previous study, and we also found that CA242 was able to distinguish colorectal cancer and gastric cancer patients from healthy controls (Table 1). The AUC values for diagnosing stage IV disease reached 0.85 or higher and, for DM, reached $0.8299(0.8117,0.8481)$ and $0.7359(0.6955,0.7764)$ in colorectal and gastric cancer patients, respectively $($ Table 2 and Table 3$)$. In patients with pancreatic cancer, cholangiocarcinoma, gallbladder carcinoma, lung cancer, cervical cancer, ovarian cancer, periampullary carcinoma, bladder cancer, breast cancer, or endometrial cancer, serum CA242 levels were positively correlated with LNM and DM (Table 2). Some diseases, such as pancreatic cancer, have been reported, but the rest are reported here for the first time. There were no significant differences in CA242 levels between patients with laryngocarcinoma, esophageal cancer, nasopharyngeal cancer, or renal cancer metastasis and those with NM nor were there different among patients with various stages of disease for these cancers (Table 2).

Among thyroid cancer, prostate cancer, and craniofacial malignant tumor patients, the level of CA242 increased with metastasis, but the differences were not significant (Table 2). While endometrial cancer and bladder cancer showed significant decreases in the sample, although the CA242 level showed an increasing trend with advancing clinical stage, the data showed no significant differences (Table 3). The remaining tumor sample sizes were too small to draw valid conclusions. According to whether the CA242 level increased with metastasis or stage advancement in the tumors listed in Tables 2 and 3 , we identified tumors as "CA242 progression-positive tumors". In these tumors, the combination of other tumor markers and CA242 could be further studied to improve the accuracy of the prediction of tumor progression. We speculate that some commonalities exist among these tumors, such as mutations in similar tumor driver genes[33], similar embryonic developmental origins, and microenvironments. Further research is needed to confirm these findings, which may have the potential to facilitate the diagnosis and treatment of tumors.

Furthermore, we also found that in all of the cancers listed in Table 4, the increase in the serum CA242 value for multiple metastases compared to NM was the largest, followed by the increases for liver metastasis and bone metastasis. Some studies have shown that the prognosis of gastric cancer with liver and bone metastasis is worse than that of any other subtype of gastric cancer, and the two organs even account for most metastatic sites [34, 35]. Interestingly, we find that similar situation also exist in colorectal cancer, lung cancer, pancreatic cancer, breast cancer and other tumors [36-40]. We deem that the liver and bones may have microenvironments conducive to the synthesis and release of CA242 molecules in the five "CA242 progression-positive tumors" or that the liver and bones enhance metastatic tumor cell growth so that more tumor cells produce more CA242, resulting in elevated levels. Therefore, CA242 may be valuable in predicting the progression of "CA242 progression-positive tumors". Moreover, studying the molecular mechanisms underlying the elevated CA242 levels may produce an understanding of the mechanisms of tumor metastasis.

However, not all malignant tumor patients' serum CA242 levels were greater than those of healthy controls. For example, the sample sizes for leukemia, laryngocarcinoma, skin cancer, and penile cancer were not small, but there were no significant differences between these patients and the healthy controls in regard to the median levels (Table 1). This finding indicated that serum CA242 levels are not suitable for diagnosing all diseases, but CA242 may be used to identify nonprimary tumors at the same site, that is, metastatic tumors with increased CA242 expression. Moreover, the median serum CA242 values of the two precancerous lesions in this study, benign colorectal neoplasms and cervical intraepithelial neoplasia, were similar to the median value of the healthy controls, and there were no significant differences. However, the median CA242 level in benign colorectal neoplasms was less than half of that in colorectal cancer, but the difference was not significant, which is probably caused by the small sample size for benign colorectal neoplasms. By increasing the number of samples, we might find a difference between these two precancerous lesions and a new marker to identify benign colorectal neoplasms and colorectal cancer, as is done for cervical cancer and cervical intraepithelial neoplasia.

This study has several limitations. First, the sample size is relatively insufficient. Although the total sample size was more than 35,000 , there were 35 different kinds of diseases, so each disease included only 1,000 patients on average. Thus, some tumors, such as colorectal cancer and liver cancer, were numerous, with more than 4,000 cases. However, some had many fewer cases, such as mediastinal tumor, ureteral cancer and fallopian tube cancer, which had only 10

Page $14 / 19$ 
cases. The reasons for this situation are not only the disparity in the incidence rates of different diseases but also the previous understanding of the diagnostic role of CA242. CA242 is considered to be a diagnostic marker for digestive system tumors[10, 13, 22, 26], especially pancreatic cancer, so measuring CA242 levels is not an appropriate choice when a doctor suspects a patient may develop other tumors. Second, in the analysis of individual diseases, the subpopulations are not sufficiently accurate. In this study, we stratified subpopulations according to the metastatic status, clinical stage, and metastatic site of the tumors. Although some differences and factors that may affect the level of CA242 were revealed, we still did not find an excellent stratified method that could make the distribution concentrated and failed to find further accurate factors that influenced CA242 levels. Third, the collection of patient clinical information is relatively incomplete, and only some patients had accurate metastasis and clinical staging information. However, we gathered no staging classification information for some tumor patients, such as those with pancreatic cancer, cholangiocarcinoma or gallbladder cancer. Therefore, we missed the opportunity for further analysis of the diseases that most commonly use CA242 in the clinic. Fourth, this was a single-center, retrospective study. Therefore, the numbers of patients in each stage and metastatic status were very different, which may lead to biased conclusions. In addition, the CA242 values for different stages and metastatic states belonged to different patients. In other words, this study had neither a paired design nor a longitudinal design so some confounding factors may have influenced the conclusion of the study.

In this study, we analyzed serum CA242 levels in 35 types of neoplastic disease patients and healthy controls and found that these levels remained unchanged in benign tumors and increased most in malignant tumors, especially pancreatic cancer, gallbladder cancer, cholangiocarcinoma, and periampullary carcinoma. The CA242 levels of these malignant digestive system tumors were significantly higher than those of other tumors and were valuable for patient diagnosis. Moreover, the level of CA242 was also related to metastasis and clinical stage and was able to predict the progression of some tumors.

\section{Conclusions}

In conclusion, CA242 can not only serve as a diagnostic biomarker for malignant digestive system tumors, such as pancreatic cancer, but also predict the progression, stage, metastasis, survival, prognosis, etc. of tumors or play a role in tumors that have not received clinical attention and are awaiting further research.

\section{Abbreviations}

AJCC

American Joint Commission on Cancer

AUC

Area under the curve

DM

Distant metastasis

LNM

Lymph node metastasis

NM

No metastasis

ROC

Receiver operating characteristic

\section{Declarations}

\section{Ethics approval and consent to participate}

This study was approved by the Ethics Committee of the First Affiliated Hospital of the Army Medical University, PLA (the approval number is KY201912), and was performed in accordance with the ethical standards laid down in the 1964 Declaration of Helsinki and its later amendments.

\section{Consent for publication}

Not applicable

\section{Availability of data and materials}

The datasets used and/or analysed during the current study are available from the corresponding author on reasonable request.

\section{Competing interests}

The authors declare that they have no competing interests

\section{Funding}

This study was supported by National Natural Science Foundation of China (Nos. 81472775, 81874211). 


\section{Authors' contributions}

ZC and KS provided the concept, HW collected the data. XQ, XS, YY and HD analysed the data. YJ and JBproofread the data and results. KS and XQ drafted the manuscript and all the authors read and approved the final manuscript.

\section{Acknowledgements}

We thank the hospital for their support of our work.

\section{References}

1. Torre LA, Bray F, Siegel RL, Ferlay J, Lortet-Tieulent J, Jemal A. Global cancer statistics, 2012. CA Cancer J Clin. 2015;65(2):87-108.

2. Lindholm L, Johansson C, Jaiisson EL, Hallberg C, Nilsson O. An inimuno-radiometric assay (IRMA) for the CA 50 antigen, Holmgren J, editor edn. Lund: Studentlitteratur; 1985.

3. Baeckstrom D, Hansson GC, Nilsson O, Johansson C, Gendler SJ, Lindholm L. Purification and characterization of a membrane-bound and a secreted mucin-typeglycoprotein carrying the carcinoma-associated sialyl-Lea epitope on distinctcore proteins. J Biol Chem. 1991;266(32):21537-47.

4. Haglund C, Lindgren J, Roberts PJ, Kuusela P, Nordling S. Tissue expression of the tumour associated antigen CA242 in benign and malignant pancreatic lesions. A comparison with CA 50 and CA 19 - 9. Br J Cancer. 1989;60(6):845-51.

5. Kuusela P, Haglund C, Roberts PJ. Comparison of a new tumour marker CA 242 with CA $19-9$, CA 50 and carcinoembryonicantigen (CEA) in digestive tract diseases. BRIT J CANCER. 1991;63(4):636-40.

6. Rothlin MA, Joller H, Largiader F. CA 242 is a new tumor marker for pancreatic cancer. CANCER-AM CANCER SOC. 1993;71(3):701-7.

7. Kawa S, Tokoo M, Hasebe O, Hayashi K, Imai H, Oguchi H, Kiyosawa K, Furuta S, Homma T. Comparative study of CA242 and CA19-9 for the diagnosis of pancreatic cancer. Br J Cancer. 1994;70(3):481-6.

8. Banfi G, Zerbi A, Pastori S, Parolini D, Di Carlo V, Bonini P. Behavior of tumor markers CA19.9, CA195, CAM43, CA242, and TPS in the diagnosisand followup of pancreatic cancer. CLIN CHEM. 1993;39(3):420-3.

9. Haglund C, Lundin J, Kuusela P, Roberts PJ. CA 242, a new tumour marker for pancreatic cancer: a comparison with CA 19 - 9 , CA 50 and CEA. BRIT J CANCER. 1994;70(3):487-92.

10. Dou H, Sun G, Zhang L. CA242 as a biomarker for pancreatic cancer and other diseases. PROG MOL BIOL TRANSL. 2019;162:229-39.

11. Tang Y, Cui Y, Zhang S, Zhang L. The sensitivity and specificity of serum glycan-based biomarkers for cancer detection. PROG MOL BIOL TRANSL. 2019;162:121-40.

12. Li X, Guo X, Li H, Lin H, Sun Y. Serum carbohydrate antigen 242 expression exerts crucial function in the diagnosis of pancreatic cancer. Tumor Biology. 2014;35(6):5281-6.

13. Gui J, Yan W, Liu X. CA19-9 and CA242 as tumor markers for the diagnosis of pancreatic cancer: a meta-analysis. CLIN EXP MED. 2014;14(2):225-33.

14. Ni XG, Bai XF, Mao YL, Shao YF, Wu JX, Shan Y, Wang J, Wang CF, Tian YT, Liu Q, et al: The clinical value of serum CEA, CA19-9, and CA242 in the diagnosis and prognosis of pancreatic cancer. European Journal of Surgical Oncology 2005, 31 (2.

15. ):164-169.

16. Bauer TM, El-Rayes BF, Li X, Hammad N, Philip PA, Shields AF, Zalupski MM, Bekaii-Saab T. Carbohydrate antigen $19-9$ is a prognostic and predictive biomarker in patients with advanced pancreatic cancer who receive gemcitabine-containing chemotherapy. CANCER-AM CANCER SOC. 2013;119(2):28592.

17. Xu H, Liu L, Xiang J, Wang W, Qi Z, Wu C, Liu C, Long J, Xu J, Ni Q, et al. Postoperative serum CEA and CA125 levels are supplementary to perioperative CA19-9 levels in predicting operative outcomes of pancreatic ductal adenocarcinoma. SURGERY. 2017;161(2):373-84.

18. Feng Z, Shi X, Zhang Q, Zhang X, Li X, Chen Z, Liu D, Sun B, Zuo Y, Ren S. Analysis of clinicopathological features and prognosis of 1315 cases in colorectal cancer located at different anatomical subsites. Pathology - Research Practice. 2019;215(10):152560.

19. You W, Sheng N, Yan L, Chen H, Gong J, He Z, Zheng K, Chen Z, Wang Y, Tan G, et al. The difference in prognosis of stage II and III colorectal cancer based on preoperative serum tumor markers. J CANCER. 2019;10(16):3757-66.

20. Li Q, Dai W, Li Y, Xu Y, Li X, Cai S. Nomograms for predicting the prognostic value of serological tumor biomarkers in colorectal cancer patients after radical resection. SCI REP-UK 2017, 7(1).

21. Ge W, Zheng L, Chen G. The Combination of Seven Preoperative Markers for Predicting Patients with Gastric Cancer to Be Either Stage IV or Non-Stage IV. GASTROENT RES PRACT. 2018;2018:1-6.

22. Wei X, Li Y, Li Y, Lin B, Shen X, Cui R, Gu Y, Gao M, Li Y, Zhang S. Prediction of Lymph Node Metastases in Gastric Cancer by Serum APE1 Expression. J CANCER. 2017;8(8):1492-7.

23. Nilsson O, Johansson C, Glimelius B, Persson B, Nørgaard-Pedersen B, Andrén-Sandberg A, Lindholm L. Sensitivity and specificity of CA242 in gastrointestinal cancer. A comparison with CEA, CA50 and CA 19 - 9. BRIT J CANCER 1992, 65(2.

24. ):215-221.

25. Banfi G, Zerbi A, Pastori S, Parolini D, Di Carlo V, Bonini P. Behavior of tumor markers CA19.9, CA195, CAM43, CA242, and TPS in the diagnosis and followup of pancreatic cancer. CLIN CHEM. 1993;39(3):420-3. 
26. Luo G, Liu C, Guo M, Cheng H, Lu Y, Jin K, Liu L, Long J, Xu J, Lu R, et al. Potential Biomarkers in Lewis Negative Patients With Pancreatic Cancer. ANN SURG. 2017;265(4):800-5.

27. Wang Y. Combined detection tumor markers for diagnosis and prognosis of gallbladder cancer. WORLD J GASTROENTERO. 2014;20(14):4085.

28. Yang X, Chen C, Peng C, Liu S, Li Y. Carbohydrate antigen 242 highly consists with carbohydrate antigen $19-9$ in diagnosis and prognosis of colorectal cancer: study on 185 cases. MED ONCOL. 2012;29(2):1030-6.

29. Zhang M, Dou H, Yang D, Shan M, Li X, Hao C, Zhang Y, Zeng P, He Y, Liu Y, et al. Retrospective analysis of glycan-related biomarkers based on clinical laboratory data in two medical centers during the past 6 years. PROG MOL BIOL TRANSL. 2019;162:141-63.

30. Byström P, Berglund A, Nygren P, Wernroth L, Johansson B, Larsson A, Einarsson R, Glimelius B. An explorative study on the clinical utility of baseline and serial serum tumour marker measurements in advanced upper gastrointestinal cancer. ONCOL REP 2010, 24(6.

31. ):1645.

32. Ni XG, Bai XF, Mao YL, Shao YF, Wu JX, Shan Y, Wang CF, Wang J, Tian YT, Liu Q, et al. The clinical value of serum CEA, CA19-9, and CA242 in the diagnosis and prognosis of pancreatic cancer. Eur J Surg Oncol. 2005;31(2):164-9.

33. Thorsen SB, Lundberg M, Villablanca A, Christensen SL, Belling KC, Nielsen BS, Knowles M, Gee N, Nielsen HJ, Brunner N, et al. Detection of serological biomarkers by proximity extension assay for detection of colorectal neoplasias in symptomatic individuals. J TRANSL MED. 2013;11:253.

34. Li F, Guo Y, Liu J, Zhang R. The significance of elevated plasma expression of microRNA 106b 25 clusters in gastric cancer. PLOS ONE. 2017;12(5):e178427.

35. Wang X, Zhang Y, Sun L, Wang S, Nie J, Zhao W, Zheng G. Evaluation of the clinical application of multiple tumor marker protein chip in the diagnostic of lung cancer. J CLIN LAB ANAL. 2018;32(8):e22565.

36. Simanshu DK, Nissley DV, McCormick F. RAS Proteins and Their Regulators in Human Disease. CELL. 2017;170(1):17-33.

37. Koo DH, Ryu M, Ryoo B, Lee S, Moon J, Chang H, Lee J, Kim TW, Kang Y. Three-week combination chemotherapy with S-1 and cisplatin as first-line treatment in patients with advanced gastric cancer: a retrospective study with 159 patients. GASTRIC CANCER. 2012;15(3):305-12.

38. Lee J, Lim T, Uhm J, Park K, Park S, Lee S, Park J, Park Y, Lim H, Sohn T, et al. Prognostic model to predict survival following first-line chemotherapy in patients with metastatic gastric adenocarcinoma. ANN ONCOL. 2007;18(5):886-91.

39. Stewart CL, Warner S, Ito K, Raoof M, Wu GX, Kessler J, Kim JY, Fong Y. Cytoreduction for colorectal metastases: liver, lung, peritoneum, lymph nodes, bone, brain. When does it palliate, prolong survival, and potentially cure? CURR PROB SURG. 2018;55(9):330-79.

40. TAMURA T, KURISHIMA K, NAKAZAWA K, KAGOHASHI K, ISHIKAWA H, SATOH H, HIZAWA N. Specific organ metastases and survival in metastatic nonsmall-cell lung cancer. Molecular and Clinical Oncology 2015, 3(1.

41. ):217-221.

42. Wu K, Tsai M, Yang C, Chang W, Hung J, Yen C, Shen C, Kuo T, Lee J, Chou S, et al. Liver metastasis predicts poorer prognosis in stage IV lung adenocarcinoma patients receiving first-line gefitinib. LUNG CANCER. 2015;88(2):187-94.

43. Kennecke H, Yerushalmi R, Woods R, Maggie CUC, Voduc D, Speers CH, Nielsen TO, Gelmon K. Metastatic Behavior of Breast Cancer Subtypes. J CLIN ONCOL. 2010;28(20):3271-7.

44. Recurrence after Resection for Ductal Adenocarcinoma of the Pancreas.

\section{Figures}



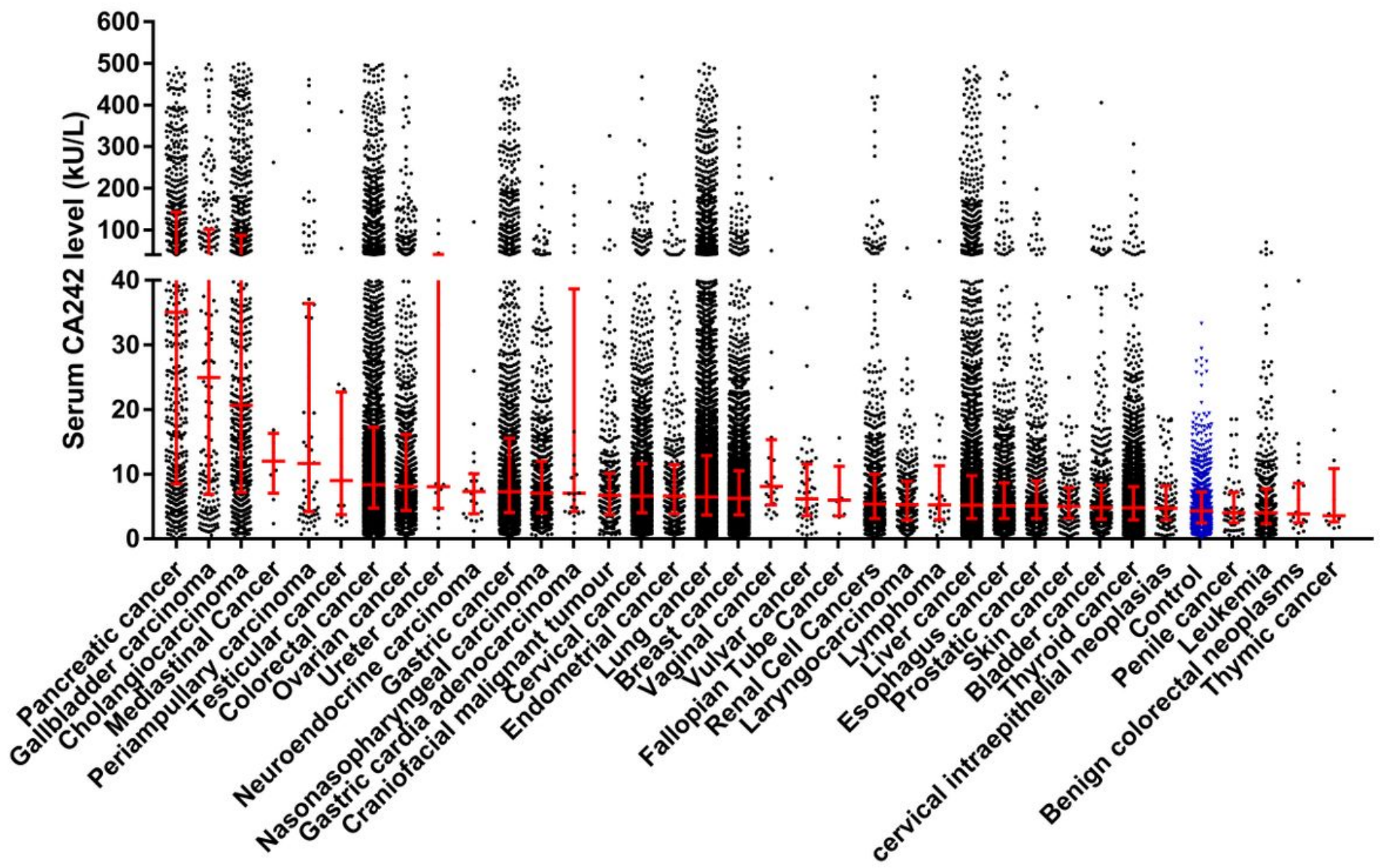

Figure 1

Serum CA242 levels in 35 different types of neoplastic diseases. The data were sorted in a descending order of the median values. CA242 levels for each group with lower quartile (25\%), median (50\%), and upper quartile $(75 \%)$ ranges were marked in red. 


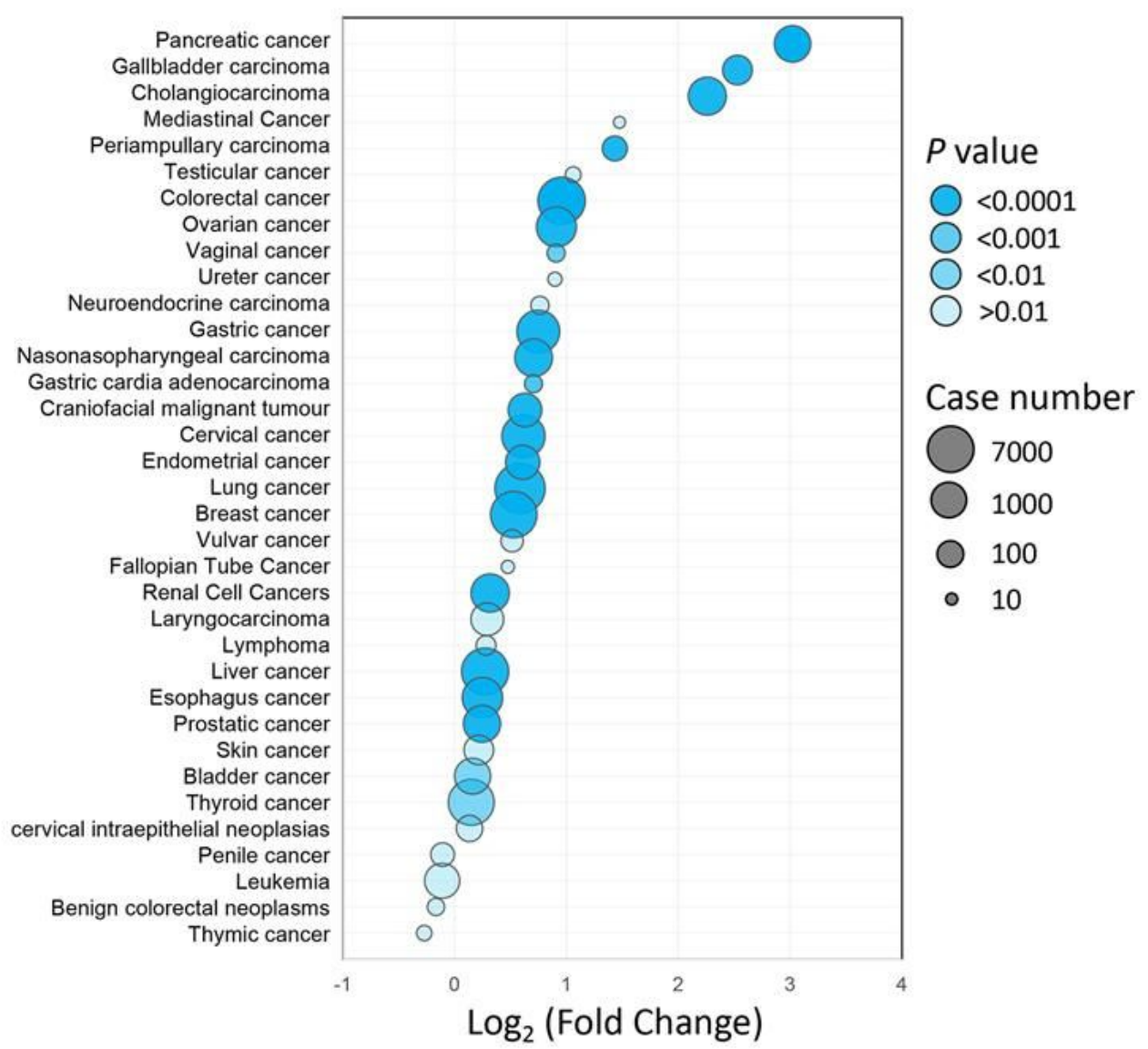

Figure 2

The relative change of serum CA242 in 35 types neoplastic disease to healthy control. Each tumor was rank in the figure according to the fold change compared with healthy control. The size of bubble was positively correlated with the case number, and the color depth was negatively correlated with the $p$ value

\section{Supplementary Files}

This is a list of supplementary files associated with this preprint. Click to download.

- SupplementalTable3.docx

- SupplementalTable2.docx

- SupplementalTable1.docx 